\title{
Inseminación artificial a tiempo fijo en diferentes genotipos y su relación con preñez en vacas en la Amazonía Ecuatoriana
}

Fixed-time artificial insemination in different genotypes and their relationship with pregnancy in cows in the Ecuadorian Amazon

López J. C. ${ }^{1,2}$., Moyano J. C. ${ }^{1,2}$, Quinteros R. ${ }^{1,2}$., Barbona I. ${ }^{1,3}$, Marini P. R. ${ }^{1,4,5}$ Daniel I.C. ${ }^{1,6}$ y Elorza P. ${ }^{6}$

${ }^{1}$ Centro Latinoamericano de Estudios de Problemáticas Lecheras (CLEPL). ${ }^{2}$ Universidad Estatal Amazónica-Centro de Investigación, Posgrado y Conservación Amazónica - Ecuador. ${ }^{3}$ Facultad de Ciencias Agrarias- UNR. ${ }^{4}$ Facultad de Ciencias Veterinarias. ${ }^{5}$ CIC-UNR. Universidad Nacional de Rosario - Argentina pmarini@unr.edu.ar. ${ }^{6}$ Universidad Veracruzana, Carretera Tuxpan-Tampico km 7.5, Colonia Universitaria, C.P.92850, Tuxpan, Veracruz. México.

Recibido: 18/02/2017

Aceptado: 30/06/2017

\section{RESUMEN}

El objetivo fue estudiar si los protocolos de inseminación artificial a tiempo fijo (IATF) y las razas están relacionados con la preñez en vacas en la Amazonia Ecuatoriana. Se evaluaron 497 registros de servicios individuales desde Octubre de 2013 a Octubre de 2014 correspondientes a vacas de diferentes razas multíparas: Brahman (B), Brown Swiss (BS), Holstein Friesian (HF) y Charolaise (Ch). Se realizó una IATF con 497 vacas aplicando dos protocolos, diferenciándose solo por el tipo de sales de estradiol utilizadas. De las 497 vacas inseminadas, 272 vacas quedaron preñadas el 55\% y 224 vacas resultaron vacías el $45 \%$ siendo $\mathrm{p} \leq 0,0001$. Los resultados encontrados entre los dos protocolos utilizados y la preñez no hubo diferencias significativas $p \geq 0,324$. De las 210 vacas utilizadas en el protocolo Benzoato de Estradiol se preñaron el $57 \%$ de las vacas y el $43 \%$ fueron vacías, con el protocolo Cipionato de Estradiol de las 286 vacas inseminadas quedaron preñadas el 53\% y el $47 \%$ de las vacas vacías. Las vacas HF fueron las que mayor porcentaje de vacías mostró independientemente del protocolo utilizado ( $28 \%$ de preñadas y $72 \%$ de vacías), con respecto al resto de las razas, (B $70 \%$ preñadas, BS $69 \%$ preñadas y las Ch $61 \%$ preñadas) en donde $p \leq 0,001$. Se concluye que no existen diferencias entre los protocolos utilizados (EC ó EB), sin embargo hay un efecto de raza en la tasa de preñez en AITF en la Amazonía Ecuatoriana.

Palabras clave: vacas, cipionato estradiol, benzoato estradiol, sistema a pastoreo tropical

\begin{abstract}
The objective was to study whether the protocols of fixed time artificial insemination and the breed are related to pregnancy in cows in the Ecuadorian Amazonia. 497 registers of individual services corresponding to different breed were evaluated from October 2013 to October 2014: Brahman (B), Brown Swiss (BS), Holstein Friesian (HF) y Charolaise (Ch). One IATF with two protocols was carried out: Out of the 497 inseminated cows, 272 got pregnant $-55 \%$ and 224 were empty $45 \%$, being $p \leq 0,0001$. The results found between the two protocols used and
\end{abstract}


pregnancy showed no significant differences $p \geq 0,324$. Out of the 210 cows used in the protocol Estradiol benzoate, $57 \%$ of cows got pregnant and $43 \%$ were empty; with the Estradiol cypionate protocol, out of 286 cows, $53 \%$ got pregnant and $47 \%$ were empty. The HF cows showed the higher percentage of empty cows, independently from the protocol being used (28\% pregnant and $72 \%$ empty), as regards the rest of the breed, (B $70 \%$ pregnant, BS $69 \%$ pregnant, Ch $61 \%$ pregnant) where $p \leq 0,001$. It is concluded that there is no differences between EC or EB synchronization protocol, however there is an effect of breed in the pregnancy rate in FTAI in the Ecuadorian Amazonia

Key words: cows, reproduction, pasture system; tropical

\section{INTRODUCCIÓN}

La producción ganadera depende de muchos factores, entre ellos el desempeño reproductivo. Un intervalo entre partos cercano al óptimo permite un mayor número de terneros nacidos y una mayor cantidad de leche producida por vaca durante la vida útil (Giovanni Báez y Henry Grajales, 2009). Los problemas reproductivos en el centro de la Amazonia Ecuatoriana, están condicionados por un sin número de factores, que directa e indirectamente conspiran para mejorar los parámetros reproductivos de la zona. Las condiciones ambientales, el manejo, la falta de aporte energético de la dieta, la salud, la infraestructura y los genotipos utilizados influyen para no lograr una eficiente reproducción. Una alternativa para mejorar los resultados, es la utilización del tratamiento hormonal tradicional para implementar una inseminación artificial a tiempo fijo (IATF), es una técnica que permite sincronizar los celos y ovulaciones de los bovinos, mediante la utilización de hormonas, lo cual hace posible inseminar una gran cantidad de animales en un corto periodo de tiempo (Raso, 2012), permite aumentar significativamente el número de animales inseminados debido a que los protocolos de (IATF) hacen posible realizar la inseminación artificial (IA) sin necesidad de detectar el celo (Cutaia, 2007; Villa et al, 2007; Bó et al, 2008). Para el mismo, se requiere del encierre de los animales en cuatro oportunidades, y uno de ellas consiste en inyectar Benzoato de Estradiol (BE) $24 \mathrm{~h}$ posteriores al retiro de los dispositivos intravaginales. La posibilidad de reducir el número de encierres y facilitar el manejo de los animales usando el Cipionato de Estradiol (CPE) aplicándolo al retirar los dispositivos es la alternativa más utilizada, ya que sincroniza las ovulaciones de manera similar a la aplicación del Benzoato de Estradiol. Sin embargo, aún en la Amazonía Ecuatoriana no hay publicaciones que muestren estos resultados obtenidos en otros ambientes en el mundo. El objetivo fue estudiar si los protocolos de inseminación artificial a tiempo fijo (IATF) y si la raza está relacionada con la preñez en vacas en la Amazonia Ecuatoriana.

\section{MATERIALES Y MÉTODOS}

Se evaluaron 497 registros de servicios individuales desde octubre de 2013 a octubre de 2014 correspondientes a vacas multíparas de diferentes genotipos: Brahman (B), Brown Swiss (BS), Holstein Friesian (HF) y Charolaise $(\mathrm{Ch})$ pertenecientes al Cantón Santa Clara, Provincia de Pastaza, Centro de la Amazonia Ecuatoriana ubicado $1^{\circ} 10$ Latitud Sur y $78^{\circ} 10$ de Longitud Oeste; $2^{\circ}$ 35 de Latitud Sur y $76^{\circ} 40$ de Longitud Oeste. El mismo se encuentra en un ambiente tropical donde la precipitación anual alcanza los $4000 \mathrm{~mm}$, la humedad relativa es del $80 \%$ y la temperatura varía entre 15 a $25{ }^{\circ} \mathrm{C}$. Su topografía se caracteriza por relieves ligeramente ondulados sin pendientes pronunciadas, distribuidos en mesetas naturales de gran extensión; la altitud varía entre los 580 y 
1200 msnm. El suelo tiene una composición muy heterogénea, sin embargo la mayoría lleva su origen desde los sedimentos fluvial procedentes desde la región andina del país. La alimentación se basó en pastoreo libre, con pastizales en base de Brachiaria decumbens (pasto Dallis), Brachiaria brizantha (pasto Marandú), Arachis pintoi (Maní forrajero), Desmodium ovalifolium y Pennisetum (Maralfalfa).

Para el ensayo se tuvieron en cuenta los siguientes criterios: (1) se utilizaron exclusivamente vacas con estado corporal $\geq$ 2,5 (Escala 1-5, Edmonsond y Lean,1989),

(2) todas las vacas estaban con 60 días de lactancia, (3) se utilizó el mismo técnico inseminador para inseminar a todas las vacas, (4) chequeos ginecológicos previo al inicio del protocolo a todas las vacas para determinar viabilidad del aparato reproductor, (5) se utilizó el mismo protocolo de descongelado (Bernardi et al., 2011), (6) el semen utilizado cumplía con los requisitos mínimos de calidad para poder ser utilizado, (7) libres de brucelosis,

tuberculosis, campilobacteriosis $\mathrm{y}$ tricomoniasis; con control de leptospirosis, rinotraqueitis infecciosa bovina y diarrea viral bovina, y aplicación del mismo plan sanitario, y confiabilidad de los datos. Se realizó una IATF con dos protocolos:

Protocolo BE: día cero se procedió a la aplicación del Progestágeno DIB- $1 \mathrm{mg}$, más $2 \mathrm{mg}$ de Benzoato de Estradiol, luego se retiró el dispositivo intra vaginal al día siete acompañado de $25 \mathrm{mg}$ de PG2F y 400 UI de eCG, y al día ocho se procedió a la aplicación de $1 \mathrm{mg}$ de Benzoato de Estradiol, y el día nueve se realizó la IATF a las 48 a 54 horas de retirado el dispositivo DIB.

Protocolo CPE. El día cero se procedió a la aplicación del Progestágeno DIB- 1 mg, más $2 \mathrm{mg}$ de Benzoato de Estradiol, se retiró el dispositivo intra vaginal al día siete acompañado de $25 \mathrm{mg}$ de PG2F y 400 UI de eCG más la aplicación de $0,5 \mathrm{mg}$ de
Cipionato de Estradiol, y al día nueve se realizó la IATF a las 48 a 54 horas de retirado el dispositivo DIB.

En el Cuadro 1 se presenta la distribución de la cantidad de vacas utilizadas por genotipo y protocolo utilizado

Cuadro 1: Distribución de los animales utilizados por protocolo y genotipos

\begin{tabular}{|l|c|c|c|c|c|}
\hline Protocolos & \multicolumn{5}{|c|}{ Genotipos } \\
\hline & B & BS & Ch & HF & Total \\
\hline BE & 29 & 73 & 58 & 51 & 211 \\
\hline CE & 37 & 102 & 47 & 100 & 286 \\
\hline Total & 66 & 175 & 105 & 151 & 497 \\
\hline
\end{tabular}

El diagnostico de preñez se hizo a los 30 días posteriores a la inseminación, a través de ecografía.

Se aplicó un modelo lineal generalizado de Regresión Logística, mediante el programa estadístico SPSS para Windows® (SPSS Inc., Chicago, IL, versión 13.0), siendo la variable respuesta considerada: "Preñez" que toma 2 valores "Preñada" o "Vacía". Las variables independientes son "Raza" (HF, Ch, BS y B) y "Protocolo" (CE y BE) siendo la expresión funcional del modelo la siguiente:

$$
(1-())={ }_{0+11+22+33+44}^{()}
$$

Donde,

$$
\begin{aligned}
& \text { ( ) es la probabilidad de que una vaca resulte preñada dado el vector. } 0 \text { es el } \\
& \text { intercepto. } \\
& 1,2 \text { y } 3 \text { los respectivos coeficientes asociados a las variables dummies ( } 1,2 \text { y } 3) \\
& \text { definidas para los genotipos y conformadas de la siguiente forma: }
\end{aligned}
$$

\begin{tabular}{|c|c|c|c|}
\hline Genotipo & & & \\
\hline B & 1 & 0 & 0 \\
\hline BS & 0 & 1 & 0 \\
\hline CH & 0 & 0 & 1 \\
\hline HF (categoría de referencia) & 0 & 0 & 0 \\
\hline
\end{tabular}


Se utilizó este tipo de modelo, ya que resultó adecuado para estudiar la relación funcional entre variables independientes y una

variable dependiente con respuesta dicotómica. En este caso, nos permitió estudiar si la raza y el protocolo afectan o están relacionadas con la preñez. La interpretación del modelo se realizó en base a las Razones de Odds estimadas.

\section{RESULTADOS}

Sin tener en cuenta los protocolos utilizados de las 497 vacas inseminadas, 272 vacas quedaron preñadas el $55 \%$ y 224 vacas resultaron vacías el $45 \%$ siendo $\mathrm{P}=496\left(\mathrm{X}^{2}\right.$ $=682,9) \quad \mathrm{p} \leq 0,0001$. De las 210 vacas utilizadas en el protocolo BE se preñaron el $57 \%$ de las vacas y el $43 \%$ fueron vacías, con el protocolo CE de las 286 vacas inseminadas quedaron preñadas el $53 \%$ y el $47 \%$ de las vacas vacías.

\section{Interpretación del Modelo de Regresión Logística.}

Se ajusta un modelo de Regresión Logística sin interacción entre las variables independientes, debido a que previamente se ajustó uno con interacción resultando ésta no significativa. Es decir, se trabaja con un modelo de efectos aditivos. Al inspeccionar la significación de las variables en el modelo, se observa que la Raza aporta significativamente al modelo (p-valor $<0.0001)$. Mientras que el protocolo no resulta significativo ( $\mathrm{p}$-valor $=0,9403$ ). En la Tabla 2 se presentan las estimaciones Máximo Verosímiles de los coeficientes del modelo y en la Tabla 3 las Razones de Odds calculadas.

Tabla 2: Estimaciones Máximo Verosímiles del los coeficientes del Modelo.

\begin{tabular}{|c|c|c|c|c|c|c|}
\hline Parámetro & & GL & Estimación & $\begin{array}{c}\text { Standard } \\
\text { Error }\end{array}$ & Wald Chi-Cuad & p-valor \\
\hline Intercepto & & 1 & -0.9494 & 0.1936 & 24.0609 & $<.0001$ \\
\hline Genotipo & $\mathrm{B}$ & 1 & 1.7758 & 0.3244 & 29.9690 & $<.0001$ \\
\hline Genotipo & $\mathrm{BS}$ & 1 & 1.7234 & 0.2446 & 49.6324 & $<.0001$ \\
\hline Genotipo & $\mathrm{Ch}$ & 1 & 1.3865 & 0.2737 & 25.6580 & $<.0001$ \\
\hline Protocolo & $\mathrm{BE}$ & 1 & 0.0148 & 0.1980 & 0.0056 & 0.9403 \\
\hline
\end{tabular}

Tabla 3: Razones de Odds Estimadas

\begin{tabular}{|l|c|c|c|}
\hline \multicolumn{1}{|c|}{ Razón de Odds } & \multirow{2}{*}{ Estimación } & \multicolumn{2}{c|}{ I.C. del 95\% } \\
\cline { 3 - 4 } & & LI & LS \\
\hline Genotipo B vs HF & 5.9051 & 3.1269 & 11.1518 \\
\hline Genotipo BS vs HF & 5.6035 & 3.4693 & 9.0508 \\
\hline Genotipo Ch vs HF & 4.0010 & 2.3397 & 6.8417 \\
\hline Genotipo B vs BS & 1.0538 & 0.5701 & 1.9481 \\
\hline Genotipo B vs Ch & 1.4759 & 0.7654 & 2.8462 \\
\hline Genotipo BS vs Ch & 1.4 .005 & 0.8425 & 2.3283 \\
\hline Protocolo BE vs CE & 1.015 & 0.689 & 1.496 \\
\hline
\end{tabular}




\section{Interpretación de las Razones de Odds:}

- La chance de preñez para un genotipo B es 5.9 veces más que la de una vaca $\mathrm{HF}$.

La chance de preñez para un genotipo BS es 5.6 veces más que la de una vaca $\mathrm{HF}$.

- La chance de preñez para un genotipo Ch es 4 veces más que la de una vaca $H F$.

La chance de preñez para un genotipo B es la misma que para uno de raza $B S$.

La chance de preñez para un genotipo B es 1.5 veces más que la de una vaca $\mathrm{Ch}$.

- La chance de preñez para un genotipo BS es 1.4 veces más que la de una vaca $\mathrm{Ch}$.

La chance de preñez para el protocolo $\mathrm{BE}$ es la misma que para el protocolo $\mathrm{CE}$.

\section{DISCUSIÓN}

Los resultados reproductivos están en coincidencia con aseveraciones de otros investigadores utilizando similares protocolos de IATF, en donde el valor registrado (BE: $57 \%$ y CPE: $53 \%$ ), se encuentran dentro del rango citado en la bibliografía que va de $43,3 \%$ a $62,7 \%$ (Chesta et al., 2009; Cutaia et al., 2007; Sales et al., 2012) y similar a lo reportado por Callejas et al., (2014), utilizando el mismo protocolo pero en vaquillonas. Los resultados de preñez también coinciden con diferentes trabajos realizados en algunas zonas de la Amazonía (Odde, 1990; Salgado et al., 2007). La técnica de inseminación y el protocolo de descongelado, deberían tenerse en cuenta para encontrar mejores porcentajes de preñez como respuestas a las IATF. La amplia diferencia en la tasa de preñez también puede deberse en parte, al nivel de ciclicidad y a la condición corporal, por tal motivo son otros factores que deben ser tomados en cuenta a la hora de decidir implementar un programa de

IATF (Humbolt et al., 1996). La comparación de los dos protocolos utilizados no mostraron diferencias significativas coincidiendo, con un trabajo de en donde el uso de CE administrado en el momento de retirar el dispositivo intravaginal con progesterona en lugar del BE permitió obtener en la mayoría de las veces similares porcentajes de preñez (Biondini et al., 2011; Callejas et al., 2014). Otros trabajos han demostrado los mismo resultados, utilizando el $\mathrm{CE}$ al retiro de los dispositivos permitiendo disminuir el número de encierres de los animales, y obteniendo porcentajes de preñez equivalentes al BE aplicado $24 \mathrm{~h}$ posteriores (MacMillan et al., 2003, Meneghetti et al., 2009). La posibilidad que ofrece el CE permitiendo disminuir un día de encierre repercutiría en varios factores que haría más eficiente el sistema desde lo económico y desde el animal. Pero a la Amazonía además, habría que tener en cuenta que reduciría en un día las dificultades que implica llegar al establecimiento.

En la Tabla 3 se observa comportamiento de una menor eficiencia reproductiva por parte de HF, en este caso esto se deba a que las $\mathrm{HF}$ poseen un genotipo más especializado en producción de leche y generalmente la recuperación del estado general para llevar adelante una gestación le lleve más tiempo que al resto, debido a las condiciones de alimentación y manejo de la Amazonía. En contraposición, las vacas $\mathrm{B}, \mathrm{BS}$ y $\mathrm{Ch}$ mostraron un mejor comportamiento reproductivo, expresando una adaptación al sistema en 
el cual vive. Estos resultados se asemejan a los reportados por RodríguezHernández et al., (1995) en donde en el trópico venezolano encontró valores menores de preñez en HF en comparación a vacas mestizas y mosaico-cebuinas. Esto demostraría la necesidad de evaluar el comportamiento de los genotipos introducidos en un ambiente diferente al cual se seleccionan los mismos. Es necesarionotomarparámetros

establecidos que podrían estar perjudicando el verdadero crecimiento de la ganadería en zona en estudio. Por lo tanto, en base a los resultados encontrados, se puede decir que la chance de preñez varía para los distintos genotipos. En cuanto a los protocolos, la chance de preñez es la misma en ambos.

\section{CONCLUSIÓN}

Se concluye que no existieron diferencias entre los protocolos utilizados (EC ó EB), sin embargo hay un efecto de raza en la tasa de preñez en AITF en la Amazonía Ecuatoriana.

\section{LITERATURA CITADA}

Alonso Villa Néstor, Morales Carlos Andrés, Granada José Fernando, Mesa Henry, Gomez Germán, Molina Juan José. 2007. Evaluación de Cuatro Protocolos de Sincronización Para Inseminación a Tiempo Fijo en Vacas Bos indicus Lactantes Evaluation of Four Synchronization Protocols for Fixed Time Artificial Insemination in Bos indicus Lactating Cows. Rev. Cient. (Maracaibo) [Internet]. 2007 Oct [citado 2016 Abr 29] ; 17( 5 ): 501-507.

Disponible en: http://www.scielo.org.ve/scielo.php?scrip $\mathrm{t}=$ sci_arttext\&pid=S0798-

22592007000500010\&lng=es.

Ambrose JD, Kastelic JP, Rajamahendran R, Aali M, Dinn N. 2005. Progesterone (CIDR)-based timed AI protocols using $\mathrm{GnRH}$, porcine LH or estradiol cypionate for dairy heifers: Ovarian and endocrine responses and pregnancy rates. Theriogenology 64: 1457-1474

https://doi.org/10.1016/j.theriogenology.2

Ayres H, Martins CM, Ferreira RM, Mello JE, Dominguez JH, Souza AH, Valentin R, Santos IC, Baruselli PS. 2008. Effect of timing of estradiol synchronization of ovulation in suckling Nelore cows (Bos indicus) treated with a progesterone-releasing intravaginal device. Anim Reprod Sci 109: 77-87. https://doi.org/10.1016/j.anireprosci.2007.

Bernardi, S., Allende, R., Mazeo, R., Monti, J. y Marini, P.R. 2011. Evaluación de los cambios ocasionados en espermatozoides bovinos por variaciones en el manejo de las dosis durante su manipulación en inseminación artificial, InVet 13 (2):25-39.

Edmonsond, A. J. y Lean, I. J. 1989. A body condition scoring chart for Holando dairy cows.Tulare 93274. Journal of Dairy Science, v. 72, p. 68-78.

https://doi.org/10.3168/jds.S0022-0302(89 Bó GA, Baruselli PS, Moreno D, Cutaia L, Caccia M, Trí bulo R. 2002. The control of follicular wave development for self appointed embryo Transfer programs in cattle. Theriogenology 57:5372.

https://doi.org/10.1016/S0093-691X(01)0

Bó GA, Baruselli PS, Martínez MF. 2003. Pattern and manipulation of follicular development in Bos indicuscattle. Anim Reprod Sci 78: 307-326.

https://doi.org/10.1016/S0378-4320(03)00 
Callejas, S.; Uslenghi, G.; Catalano, R.; Larghi, J.; Cabodevila, J 2014. Comparación de dos protocolos para sincronizar ovulación e implementar inseminación artificial en vaquillonas. Rev vet 25(2): 100-104. https://doi.org/10.30972/vet.252502

Cutaia LE, Peres LC, Pincinato D, Chesta PM, Ramos M, Bo G. 2007. Programas de sincronización de celos en vaquillonas de carne: puntos críticos a tener en cuenta. Anales VII Simp Intern Reprod Anim, Córdoba, Argentina, p. 83-104

Chesta P, Brandan A, Cuestas G, Quiñones G, Lozano P, Tríbulo H. 2009. Evaluación de diferentes dosis de cipionato De estradiol sobre la tasa de preñez en inseminación artificial a tiempo fijo en vaquillonas de 15 meses de edad. Anales VIII Simp Intern Reprod Anim IRAC, Córdoba (Argentina), CD N 13.

Chesta P, Filippi L, Ramos M, Racca D, Bó G. 2009. Evaluación de las tasas de preñez en protocolos de inseminación Artificial a tiempo fijo (IATF) utilizando diferentes dosis de cipionato de estradiol en vaquillonas Holando. Anales VII Simposio Internacional de Reproducción AnimalIRAC, Córdoba, Argentina, CD $\mathrm{n}^{\mathrm{o}} 12$.

Bó GA, Adams GP, Pierson RA, Mapletoft RJ. 1995. Exogenous control of follicular wave emergence in cattle. Theriogenology 43: 31-40.

https://doi.org/10.1016/0093-691X(94)000

Bó GA, Baruselli PS, Moreno D, Cutaia L, Caccia M, Tríbulo R. 2002. The control of follicular wave development for self appointed embryo transfer programs in cattle. Theriogenology 57: 53-72.

https://doi.org/10.1016/S0093-691X(01)0

Bó GA, Baruselli PS, Martínez MF. 2003. Pattern and manipulation of follicular development in Bos indicus cattle. Anim Reprod Sci 78: 307-326.

https://doi.org/10.1016/S0378-4320(03)00 Bó, G. A., Cutaia, L. E., Souza, A. H., \& Baruselli, P. S. (2008).Actualización sobre protocolos de IATF en bovinos de leche. In Proceedings of the 3th International Symposium on Animal Reproduction Applied. Londrina, Brazil (pp. 95-110)

Colazo MG, Kastelic JP, Martinez MF, Whittaker PR, Wilde R, Ambrose JD, Corbett R, Mapletoft RJ. 2004. Fertility following fixed time AI in CIDR-treated beef heifers given GnRH or estradiol cypionate and fed diets supplemented with flax seed or sunflower seed. Theriogenology 61: 1115-1124

https://doi.org/10.1016/j.theriogenology.2

Giovanni Báez S. and Henry Grajales L., 2009. Anestro posparto en ganado bovino en el trópico. Rev.MVZ Córdoba 14(3):1867-1875.

https://doi.org/10.21897/rmvz.347

Humbolt, P., Grimard, B., Mialot, J. 1996 Sources of variation of post-partum cyclicity, ovulation and pregnancy rates in suckled beef cows treated with progestagen and PMSG. Proceedings Society for Theriogenology Meeting, Kansas City; $36-45$.

MacMillan KL, Segwagwe BE, Pino CS. 2003. Associations between the manipulation of patterns of follicular development and fertility in cattle. Anim Reprod Sci 78: 327-344.

https://doi.org/10.1016/S0378-4320(03)00 Meneghetti M, Sá-Filho OG, Peres RF, Lamb GC, Vasconcelos JL. 2009. Fixed time artificial insemination with estradiol and progesterone for Bos indicus cows I: Basis for development of protocols. Theriogenology 72: 179-189.

https://doi.org/10.1016/j.theriogenology.2 
Odde, K.G. 1990. Synchronization of estrus in postpartum cattle. J.Anim. Sci. 68:817-830

https://doi.org/10.2527/1990.683817x

Raso, M. (2012). Inseminación artificial a tiempo fija (IATF).Ganadería. Carpeta Técnica EEA Esquel pág 203-205.

Roger Salgado O, Marco González T, Juan Simanca 2007. Inseminación artificial a tiempo fijo en vacas Brahman lactantes. Rev. MVZ Córdoba 12(2): 1050-1053

https://doi.org/10.21897/rmvz.426

Rodríguez-Hernández T., Espinoza J., y Verde, O. 1995. Efecto del momento de inseminación artificial, masaje clitórico, temperatura rectal y otros factores sobre la preñez en bovinos. Zootecnia Trop. Vol. 13(2):129-149.

Sales JN, Carvalho JB, Crepaldi GA, Cipriano RS, Jacomini JO, Maio JR, Souza JC, Nogueira GP, Baruselli PS. 2012. Effects of Two estradiol esters (benzoate and cypionate) on the induction of synchronized ovulations in Bos indicus cows submitted to a timed artificial insemination protocol. Theriogenolog 78:510-516.

https://doi.org/10.1016/j.theriogenology.2 
Copyright (c) 2017 J. C. López, J. C. Moyano, R. Quinteros, I. Barbona, P. R. Marini,

Daniel I.C. y Elorza P.

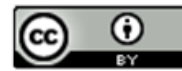

Este tex to está protegido por una licencia licencia Creative Commons $\underline{4.0}$

Usted es libre para Compartir —copiar y redistribuir el material en cualquier medio o form ato- y Adaptar el documento —remezclar, transformar y crear a partir del material- para cualquier propósito, , incluso para fines comerciales, siempre que cumpla la condición de:

Atribución: Usted debe dar crédito a la obra original de manera adecuada, proporcionar un enlace a la licencia, e indicar si se han realizado cam bios. Puede hacerlo en cualquier form a razonable, pero no de form a tal que sugiera que tiene el apoyo del licenciante o 10 recibe por el uso que hace de la obra.

Resumencielicencia - Textocompletodelalicencia 